\title{
Three-Dimensional Motion Analysis of Mitral Valve Leaflet in Children with Congenital Heart Disease and Its Clinical Significance: A Pilot Study
}

\author{
K Sun ${ }^{1}, \mathrm{X} \mathrm{Yan}^{2}, \mathrm{LP} \mathrm{Yao}^{1}, \mathrm{Q} \mathrm{Guo}^{2}$, \\ LP Wu ${ }^{1}$, YF Shang ${ }^{2}$ \\ ${ }^{1}$ Shanghai Children's Medical Center,XinHua Hospital, Affiliated to Medical School of Shanghai \\ JiaoTong University, Shanghai PR China \\ ${ }^{2}$ Institute of Image Processing \& Pattern Recognition, Shanghai Jiaotong University,Shanghai PR
} China

\begin{abstract}
This study is to quantitatively evaluate abnormal movement of mitral apparatus (MA) in patient with congenital mitral valve regurgitation using self-designed three dimensional motion analyses software and enable doctors to intuitionistic and "en-face" visualize the mitral valve's movement by using pseudocolor coding of the moving velocity of mitral valve leaflet. Initial evaluation of the software was conducted in 20 children with congenital mitral valve regurgitation (MR) including 10 children with mild MR and 10 children with moderate to severe MR. Results showed that, the moving velocity of the rough zone of mitral valve during systolic phase in children with moderate to severe MR is much high than that in control group. It's indicated that three dimensional motion analysis of mitral valve could emerge to be a new potential useful parameter for evaluation the truly mechanism of $M R$.
\end{abstract}

\section{Introduction}

The mitral apparatus is made up of mitral annulus, valve, chords and papillary muscles. It is the most complex and finest apparatus in the heart. At presents, the three-dimensional echocardiographic study of mitral apparatus only restricted in morphology observation[13].Few report has concerned about quantitative evaluating of the three dimensional spatial objects of mitral apparatus.

\section{Methods}

The self-designed three dimensional motion analyses software was developed to enable doctors to intuitionistic and "en-face" visualize the mitral valve's movement by using pseudocolor coding of the moving velocity of mitral valve leaflet, which including five modules: data set format convert, noise filter, interpolation, three dimensional reconstruction and motion analysis as following :

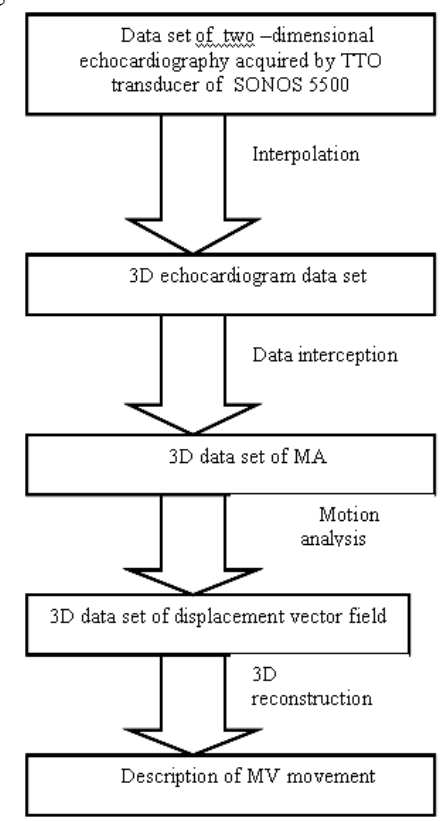

Initial evaluation of the software was conduct in 20 children with congenital mitral valve regurgitation (MR) including 10 children with mild MR and 10 children with moderate to severe MR. Twenty age and sex matched children was selected as control group.

\section{Results}

There is no different in mitral valve movement velocity between the of group of patients with mild MR and control group. And, the same result could be found between the group of children with moderate to severe MR and control group during diastole. But, the moving velocity of the rough zone of mitral valve during systolic phase in children with moderate to severe MR is much 
high than that in control group (Fig.1,2).
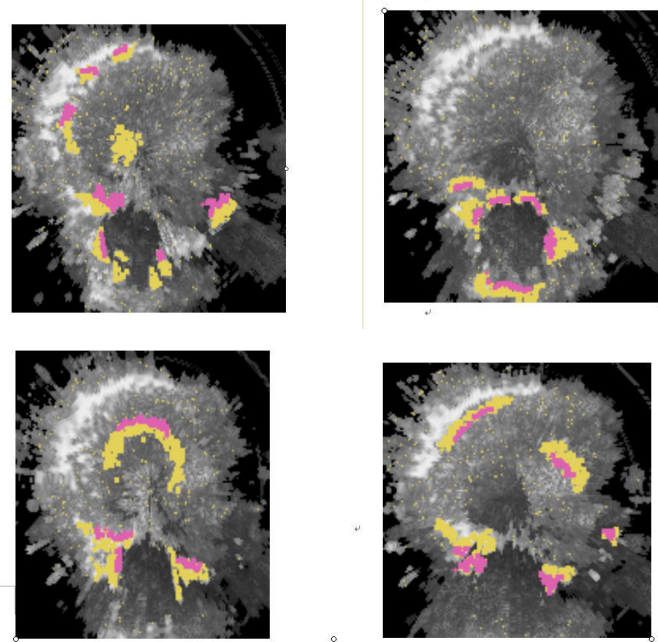

Figure 1. Enface view of MV from Left atrium showing that there is no significant difference of MA motion between children with normal MV (right column) and with mild MR (left column ) during systole(upper row ) and diastole (lower row )phases.
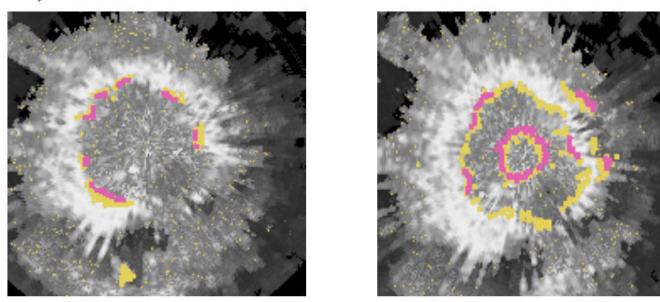

Figure 2. This picture depicts the differentiation of MV movement during systole of cardiac cycle, which appears that the moving velocity of the rough zone of mitral valve during systolic phase in children with moderate to severe MR (left) is much high than that in control children (right ) .

\section{Discussion and conclusions}

It's indicated that three dimensional motion analysis of mitral valve could emerge to be a new potential useful parameter for evaluation the truly mechanism of MR and provide a useful hint for successful mitral valve repair. Further studies, including analysis of three dimensional mitral valve motion curve during the heart cycle and the relationship between each specific anomaly of mitral valve apparatus and the change of the value of mitral valve motion, which might be very useful in the operation plant design for patients with mitral valve regurgitation, are necessary in the future.

\section{Acknowledgements}

This work was partially supported by National Basic Research Program of China (No.2003CB716104) and Shanghai Science and Technology Development Foundation (034119820).

\section{References}

[1] Fabricius AM, Walther T, Falk V. Three-Dimensional Echocardiography for Planning of Mitral Valve Surgery: Current Applicability? Ann Thorac Surg 2004;78:575- 578

[2] Qin JX, Shiota T, Tsujino T et al. Mitral annular motion as a surrogate for left ventricular ejection fraction: real-time three-dimensional echocardiography and magnetic resonance imaging studies Eur J Echocardiography 2004: 5, 407-415

[3] Salustri A, Becker AE, van Herwerden L et al. Threedimensional echocardiography of normal and pathologic mitral valve: a comparison with two-dimensional transesophageal echocardiography.J Am Coll Cardiol 1996;27:1502-1510.

[4] Chauvel C, Bogino E, Clerc P, Fernandez G et al. Usefulness of three-dimensional echocardiography for the evaluation of mitral valve prolapse: An intraoperative study. J Heart Valve Dis 2000;9:341-349.

[5] Sutaria N, Northridge D, Masani $N$ et al. Three dimensional echocardiography for the assessment of mitral

[6] valve disease. Heart 2000;84(suppl II):7-10.

Address for correspondence

Name Kun Sun

Full postal address 1678 DongFang Rd. Shanghai 200433 PR China

E-mail address sunkun@sh163.net 